\title{
Erfahrungsberichte aus entwicklungspolitischen Freiwilligendiensten: Bücher als Medium für die entwicklungspolitische Bildungsarbeit?
}

\author{
Buffy Katharina Löhr \\ MSc Political Economy of Violence, Conflict and Development \\ Internationale Jugendgemeinschaftsdienste e.V. (ijgd), Bereich \\ Freiwilligendienste in Afrika, Asien und Lateinamerika | Pädagogische Referentin \\ für Freiwilligendienste in Afrika | buffy.loehr@ijgd.de \\ Schlagwörter: Freiwilligendienst, Berichterstattung, Reiseberichte, Rückkehrendenarbeit
}

Die Rückkehrarbeit ist ein zentrales Element des entwicklungspolitischen Freiwilligendienstes weltwärts. ${ }^{3}$ Freiwillige sollen ihre Erfahrungen in die Gesellschaft tragen und über den Auslandseinsatz hinaus einen persönlichen Beitrag für eine gerechtere Welt leisten ${ }^{4}$. Es gibt zahlreiche Möglichkeiten während und nach dem Freiwilligendienst über die eigenen Erfahrungen zu berichten und sich gesellschaftlich einzubringen. Engagement von Rückkehrenden bei Entsendeorganisationen im Rahmen der Vor- und Nachbereitung von zukünftigen Freiwilligen, Vorträge an Schulen, in Gemeinden und in privaten Kreisen, sowie Engagement bei diversen Initiativen und Organisationen sind weit verbreitet. Darüber hinaus ist ein neues Phänomen beobachtbar, das an Popularität zu gewinnen scheint: Zunehmend veröffentlichen Freiwillige ihre Erfahrungen auch als Buch (z. B.: Kamp 2012; Walter 2012; Dern 2013; Lombardo 2013; Müller 2013). In diesem Beitrag wird der Nutzen dieser Publikationen für Entsendeorganisationen erörtert.

Aktuelle Publikationen von Freiwilligen erwecken den Eindruck, als ob von Verlagsseite ein hohes Interesse an Auslandberichten besteht; unabhängig von Dauer oder Format des Dienstes. So ruft bspw. der Verlag „interconnections“ in seinen Publikationen mehrfach zur Einsendung von Manuskripten zur Veröffentlichung auf. Ein Autor (Walter 2012) berichtet, dass der Verlag ihm die Veröffentlichung seines Blogs vorgeschlagen hat. Es ist unklar, woher das anscheinend gestiegene Interesse an Erfahrungsberichten von Freiwilligen kommt und mit welchem Zweck Berichte veröffentlicht werden.

Die Veröffentlichung der eigenen Erfahrungen in Form von Büchern kann dem Programmziel von weltwärts entsprechen. Es stellen sich jedoch die Fragen, welchen Beitrag die Rückkehrenden mit ihren Büchern leisten und für wen diese

\footnotetext{
3 Weltwärts ist der entwicklungspolitische Freiwilligendienst in Deutschland, der seit 2008 vom Bundesministerium für wirtschaftliche Zusammenarbeit und Entwicklung gefördert wird. Etwa 200 zivilgesellschaftliche Entsendeorganisationen entsenden über das Programm jährlich zwischen 3000 und 4000 Freiwillige in Länder des globalen Südens.

4 http://www.weltwaerts.de/programm.html
} 
Publikationen von Interesse sein können. Neben Familienangehörigen, Freunden und Bekannten der Rückkehrenden, könnten zukünftige Freiwillige eine potentielle Leserschaft sein und darüber hinaus Entsendeorganisationen Interesse an den Erfahrungsberichten für die Verwendung in der eigenen pädagogischen Bildungsarbeit haben. Es wäre denkbar, dass Entsendeorganisationen Freiwilligen, die in dasselbe Gastland gehen, die jeweilige Publikation zur Vorbereitung empfehlen. Auch könnten die Organisationen Auszüge aus den Publikationen verwenden, um diese z. B. als Diskussionsgrundlage oder Erfahrungsbericht in der Vorbereitung einzubinden. Dies wäre eine Möglichkeit für Entsendeorganisationen, die keine Rückkehrenden persönlich in die Vorbereitung einbeziehen, Freiwilligenperspektiven zu integrieren, um Inhalte anschaulicher zu machen. Und auch wenn Organisationen beginnen neue Partnerschaften aufzubauen und noch keine Erfahrungsberichte zu den Kooperationsländern haben, könnten die Publikationen hilfreich sein.

Um den Wert der Publikationen für Entsendeorganisationen näher zu bestimmen, werden folgend drei kürzlich veröffentlichte Publikationen von Freiwilligen besprochen. Es handelt sich um Veröffentlichungen, die alle über einen Freiwilligendienst in einem afrikanischen Land berichten und von weltwärts-Freiwilligen geschrieben wurden. Bei der folgenden Besprechung gilt zu beachten, dass Berichte von Auslandsaufenthalten, insbesondere Blogs, bereits vielfach macht- und rassismuskritisch betrachtet wurden (z.B. Kontzi 2013; Bendix/Danielzik 2013; Stark/Weber 2012; glokal 2012). Fragen und Erkenntnisse dieser Publikationen treffen auch auf die veröffentlichten Bücher zu. Sie werden in diesem Beitrag nicht im Detail wiederholt, prägen jedoch die folgende Auseinandersetzung.

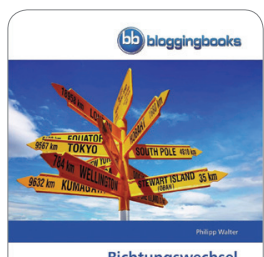

Richtungswechsel Ein weltwairts-Freiwiliger erzbiht von seiren
Erichnisson und Ertahrungon in Namibla
Der Freiwillige Phillip Walter (2012) nennt als Ziel seiner Publikation, keinen weiteren Reiseführer zu Namibia erstellen, sondern einen subjektiven Erlebnisbericht teilen zu wollen. Positiv ist, dass er seinen Blogeinträgen kurze Informationen zu seiner Einsatzstelle, dem Ort des Freiwilligendienstes und dem weltwärts-Programm, voran stellt und auch Kritik benennt, die gegenüber dem Programm geäußert wird. Weiterhin gibt es nach jedem Quartal ein kurzes Zwischenfazit. Dennoch liest sich sein Buch primär wie ein Reisebericht, bei dem Lesende viel über seine Unternehmungen am Wochenende und in den Ferien erfahren, sowie Detailinformationen zu Geldpreisen und dem Konsum von Alkohol. Er gibt nur sehr begrenzt Einblicke in sein Alltagsleben, die direkte Freiwilligentätigkeit oder auch das gemeinsame Leben und Arbeiten mit der namibischen Bevölkerung. Während Walter die Vielfalt Afrikas zeigen will, ist das Buch geprägt von Stereotypen, Pauschalisierungen und dem Wunsch im Projekt strukturelle Veränderungen zu schaffen. Im Norden des Landes findet er 
das ,wahre Afrika', definiert durch viele Menschen, hohes Verkehrsaufkommen, Märkte und Hitze. Er feiert in Clubs, die auf Grund der Aufmachung Europa zugeordnet werden. Seine Schwierigkeit, soziale Beziehungen aufzubauen, liegt laut Walter an der Mentalität der Hereros. Vor Abreise ist es noch sein Ziel in wenigen Wochen den Schüler_innen in seinem Projekt das Lernen von Vokabeln mit Karteikartenkästen aus Deutschland beizubringen, damit nachfolgende Freiwillige weniger Schwierigkeiten haben und eine Routine bei der Umsetzung der neu eingeführten Lehrpläne zu erreichen. Die Idee, am Ende des Buches Zusatzinformationen über das Land hinzufügen, ist begrüßenswert, jedoch ist die geschichtliche Darstellung der Kolonialzeit und des Genozids in Namibia leider sehr komprimiert und damit oberflächlich.

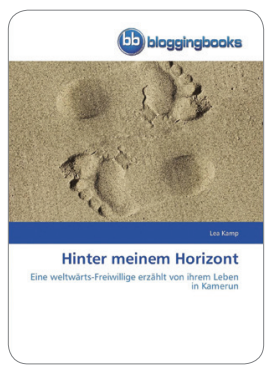

Lea Kamp (2012) hat ebenfalls ihre Blogeinträge zum Freiwilligendienst in Kamerun chronologisch in Buchform veröffentlicht und berichtet in diesen über den Ablauf ihres gesamten Freiwilligendienstes, inklusive des pädagogischen Begleitprogramms und individuellen Hoch- und Tiefphasen. Auch hat sie kurze Informationen zur Geschichte von Kamerun integriert, wobei auch in diesem Buch wenig Bezug zur Kolonialvergangenheit und der Rolle Deutschlands genommen wird. Auch wenn das Buch von Kamp die diversen Aspekte des Freiwilligendienstes stärker hausstellt als Walter und nicht primär die Freizeit und den Urlaub, so bringt auch ihr Bericht Fragen mit sich. Nach bereits fünf Wochen meint sie ihrerseits alle Vorurteile abgebaut zu haben und bittet ihre Leserschaft um Stereotype, um dazu einen Blogeintrag schreiben zu können. Es wird teilweise sehr wertend berichtet und Namen von Kolleg_innen, die Kamps Ansicht nach unzureichend arbeiten, werden offen genannt. Auch Kamp findet auf Reisen das ,stereotype Afrika' und schreibt viel in den vergleichenden Kategorien: Deutschland-Kamerun.

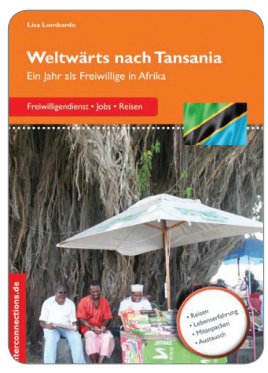

Lisa Lombardo (2013), die in Tansania war, hat sich entschieden, in regelmäßigen Abständen die im Freiwilligendienst erstellten Blogeinträge mit Gedanken nach der Rückkehr abzuwechseln. Bewusst entscheidet sie sich ihre Blogeinträge zu veröffentlichen, auch wenn sie heute diese so nicht mehr schreiben würde. Lombardo nutzt das Vorwort, um die Struktur des Buches zu erläutern, sowie eine Begriffsdefinition von „Schwarz" und „Weiß“" vorzunehmen und auf ihre Subjektivität hinzuweisen. Trotz bewusster Entscheidung der Autorin bleibt es fraglich, ob Blogeinträge, die eine Autorin auf Grund von Rassismen und Stereotypen nicht wieder so schreiben würde, reproduziert werden müssen und welchen Mehrwert diese Strategie hat. Ein Bericht ausschließlich aus 
der reflektierteren Rückkehrenden-Perspektive hätte, insbesondere in Anbetracht der Fülle von vergleichbaren Blogeinträgen, eine willkommene Abwechslung und Bereicherung sein können. Auch wenn farblich versucht wurde das Lesen zu erleichtern, so ist inhaltlich nicht immer erkennbar, welche Textpassage Blogeintrag und welche Reflexionsebene ist. So werden im Reflexionsteil weiterhin pauschalisierende Kategorien verwendet und Deutschland und Tansania bleiben in sich meist homogene und gegenseitig konträre Einheiten. Sehr bedauernswert ist, dass die die Publikation von Lombardo relativ abrupt endet - nämlich nach zwei Dritteln des Freiwilligendienstes; dem Zeitpunkt ab dem sie meint Tansania richtig kennenlernen zu können, weil sie neue soziale Zugänge findet. Diese Entscheidung stellt den Sinn des Buches stark in Frage. Sofern das Ziel im Sinne der entwicklungspolitischen Rückkehrenden-Komponente ist, Wissen über Tansania in Deutschland zu erweitern, dann wäre es wünschenswert, wenn das Buch auch dann weiter berichtet, wenn die Freiwillige im Land angekommen ist. Auch wäre es wünschenswert gewesen, neben subjektiven Blogeinträgen Informationen zum Gastland, wie z. B. Politik und Geschichte inklusive der deutschen Kolonialvergangenheit zu berücksichtigen.

Alle der hier vorgestellten veröffentlichten Erfahrungsberichte sind ausschließlich aus Perspektive der Freiwilligen geschrieben und auf Deutsch veröffentlicht. Es kann argumentiert werden, dass weltwärts ein Programm ist, welches nach Rückkehr der Freiwilligen primär das entwicklungspolitische Bewusstsein der Teilnehmenden in Deutschland verbessern soll. Allerdings wird dadurch gefördert, dass über Länder und Personen geschrieben wird, ohne dass meist genannte Personen und andere Akteure in den Aufnahmeländern auf Grund von Sprache und Zugangsmöglichkeiten die Berichte selber lesen können. Projektmitarbeiter_innen, Angehörige von Gastfamilien und zahlreiche andere Personen werden namentlich benannt, beschrieben und auch oft bewertet, ohne dass diese Personen die Möglichkeit haben die Schilderungen zu kommentieren. Weiter wird nichtdeutschsprachigen Personen in den Gastländern die Option genommen, ebenfalls Einblicke in die Gedanken und Erfahrungen der Freiwilligen zu erhalten, welches im Sinne des interkulturellen Dialoges auch ein Ziel des weltwärts-Programms sein sollte. Darüber hinaus werden ausschließlich Stimmen von Freiwilligen aus Deutschland gefördert, die über andere Länder berichten. Menschen im Globalen Süden wird in diesen Veröffentlichungen keine Plattform eröffnet, selber über ihr Land, entwicklungspolitische Zusammenhänge oder die Erfahrungen mit Freiwilligen zu berichten.

Die drei besprochenen Publikationen werden für die pädagogische Bildungsarbeit von Entsendeorganisationen nicht zusätzlich benötigt. Es handelt sich um Darstellungen, die bei Bedarf zahlreich im Internet gefunden werden können. Während Kamp und Walter fast ausschließlich ihre Blogeinträge unverändert veröffentlicht 
haben, kommen bei Lombardo zumindest die Reflexionsabschnitte hinzu. Dennoch handelt es sich bei den Publikationen primär um Erfahrungsberichte, die sich nicht unterscheiden von den stark verbreiteten Blogs von Freiwilligen, die meist öffentlich (d. h. nicht Passwort geschützt) interessierten Personen offen stehen und bei Interesse auch von Entsendeorganisationen genutzt werden können.

Auch bietet sich bei der Verwendung der Publikationen in der Vorbereitung nicht die Möglichkeit, mit den Autor_innen in Austausch zu treten, um Rückfragen zu stellen oder Eindrücke zu kontextualisieren. Sofern Entsendeorganisationen die Möglichkeiten haben, sollte auf die Verwendung dieser Bücher verzichtet werden und eher Rückkehrer_innen, sowie Vertreter_innen der Partnerorganisationen und/oder Länderreferent_innen in die Vorbereitung und pädagogische Begleitung einbezogen werden, sowie Publikationen einer diverseren Autor_innenschaft.

Interessierten Personen und all jene, die überlegen eigene Erfahrungsberichte zu veröffentlichen, sollten vor und während des Verfassens ihrer Beiträge die oben genannten Publikationen zu Berichterstattung oder auch andere (z. B. BER 2010; Arndt/Ofuatey-Alazard 2011; Arndt/Hornscheid 2004) zur Unterstützung hinzuziehen. Auch Entsendeorganisationen sollten diese Materialien in ihre Arbeit, insbesondere die pädagogische Begleitung und Rückkehrarbeit, integrieren, um die Hinweise zur Berichterstattung gemeinsam mit zukünftigen Freiwilligen zu diskutieren und Reflexionsprozesse bei den Freiwilligen und den Entsendeorganisationen zu fördern.

Abschließend bleibt unklar, woher das scheinbar gestiegene Interesse von Verlagen an Freiwilligenberichten kommt. Es eröffnet jedoch Rückkehrenden eine Plattform Berichte zu veröffentlich, ohne dass jene Verlage anscheinend Inhalte und Darstellungsweisen hinterfragen. Dieses kann einen häufig von Rückkehrenden gefühlten Experten_innen Status stärken und kontraproduktiv gegenüber dem weltwärts-Ziel sein, entwicklungspolitisches Bewusstsein zu fördern und zu einer gerechteren Welt beizutragen. Bei Blogs überwiegt trotz ihrer meist öffentlichen Zugänglichkeit der subjektive, tagebuchartige und private Charakter des Dargestellten (da die oft für sich selbst, Freunde und Verwandte, nicht aber für die allgemeine Öffentlichkeit geschrieben werden). Verlage müssen sich jedoch bewusst sein, dass der Status des Geäußerten sich durch die Veröffentlichung in Buchform ändert und tragen daher eine editorische Verantwortung, um die subjektiven Erlebnisausdrücke der Freiwilligen entsprechend einzuordnen. Auch Verlagshäuser sollten Verantwortung übernehmen, in dem sie sich mit macht- und rassismuskritischen Fragestellungen auseinandersetzen bevor sie Erfahrungsberichte publizieren. Nicht nur Freiwillige aus Deutschland sollten zu Wort kommen, sondern ebenso andere Akteure mit denen die Freiwilligen während des Auslandsaufenthaltes in Kontakt kommen. Weiter wäre es wünschenswert, dass Publikationen 
mindestens zweisprachig veröffentlicht werden, so dass erweitere Personengruppen diese lesen und kommentieren können.

Alle Entsendeorganisationen sollten im Rahmen der pädagogischen Bildungsarbeit, von der Vorbereitung bis zum Ende der Nachbereitung, mit den Freiwilligen zu den Themen Sprache und Berichterstattung arbeiten und parallel auch sich selber und die eigenen Arbeits- und Darstellungsweisen hinterfragen. Die Organisationen sollten in der Programmumsetzung Räume bieten, um Ideen zur Berichterstattung zu entwickeln und Projekte umzusetzen, um so eine differenziertere und diversifiziertere Rückkehrenden-Arbeit zu fördern. Fehlt dieser Raum für Austausch, sind Freiwillige alleine gelassen in der Zielsetzung einen gesellschaftlichen Beitrag zu leisten. Hierbei ist auch die Abstimmung mit den Partnerorganisationen unabdingbar, da diese die Freiwilligen im Ausland begleiten.

Freiwillige, die sich dafür entscheiden Berichte zu veröffentlichen, sollten sich zum einen fragen was ihr Ziel der Veröffentlichung ist. Zum anderen benötigt es der intensiven Auseinandersetzung mit der Art und Umsetzung des Vorhabens, sowie möglichst Ideen über einen individuellen Blog hinausgehend. Freiwillige könnten z. B. Beiträge unterschiedlicher Personen, mit denen sie im Freiwilligendienst zu tun haben, sammeln und bei Einverständnis der Personen auf ihrem Blog oder in einer Publikation nach Rückkehr veröffentlichen. Oder Freiwillige suchen gemeinsam mit einer Bezugsperson vor Ort eine bestimmte Begebenheit oder Gegenstand, über den unterschiedliche Personen aus verschiedenen Blickwinkeln berichten. Auch wäre denkbar, dass Freiwillige mit den Partnerorganisationen einen gemeinsamen Blog erstellen, der von allen Freiwilligendienst-Akteuren gemeinsam genutzt wird und dessen Administrationsrechte z. B. bei der Partnerorganisation liegen, so dass dieser zu einer kontinuierlichen Plattform des Dialogs wird.

Zusätzlich bietet das Süd-Nord-Programm, durch welches seit 2014 auch Freiwillige aus Ländern des Globalen Südens einen weltwärts-Freiwilligendienst in Deutschland leisten können, die große Chance, dass Berichterstattungen diverser werden und spannende neue Dialogprojekte entstehen.

\section{Literaturverzeichnis}

Arndt, Susan und Antje Hornscheid (Hrsg.) (2004): Afrika und die deutsche Sprache: Ein kritische Nachschlagewerk, Münster.

Arndt, Susan und Nadja Ofuatey-Alazard (Hrsg.) (2011): Wie Rassismus aus Wörtern spricht: (K)Erben des Kolonialismus im Wissensarchiv deutsche Sprache. Ein kritisches Nachschlagewerk, Münster.

Bendix, Daniel und Chandra-Milena Danielzik (2013): Bacardi-Feeling und Entwicklungszusammenarbeit - Zum Zusammenhang von Exotismus und Entwicklungszusammenarbeit. In Berliner Entwicklungspolitischer Ratschlag (Hrsg.), Develop-mental Turn: Neue Beiträge zu einer rassismuskritischen entwicklungspolitischen Bildungs- und Projektarbeit, Berlin. 
Berliner Entwicklungspolitischer Ratschlag (BER) (2010): Checklisten zur Vermeidung von Rassismus in der entwicklungspolitischen Bildungsarbeit, Berlin.

Dern, Sebastian (2013): Freiwilligendienst in Peking: Ein Soziales Jahr in China, Reihe Jobs, Praktika, Studium Bd. 59, interconnections Verlag, Freiburg.

glokal (2012): Mit kolonialen Grüßen...: Berichte und Erzählungen von Auslandsaufenthalten rassismuskritisch betrachtet, glokal e.V., Berlin.

Kamp, Lea (2012): Hinter meinem Horizont: Eine weltwärts-Freiwillige erzählt von ihrem Leben in Kamerun, Bloggingbooks, Saarbrücken.

Kontzi, Kristina (2013): Die Freiwillige ist Königin - Ein machtkritischer Blick auf das Freiwilligen-Programm „weltwärts“. In Berliner Entwicklungspolitischer Ratschlag (Hrsg.), Develop-mental Turn: Neue Beiträge zu einer rassismuskritischen entwicklungspolitischen Bildungs- und Projektarbeit, BER, Berlin.

Lombardo, Lisa (2013): weltwärts nach Tanzania: Ein Jahr als Freiwillige in Afrika, Reihe Jobs, Praktika, Studium Bd. 61, interconnections Verlag, Freiburg.

Müller, Konrad (2013): Freiwilligendienst in Gambia: Engagement in einer fremden Welt, Reihe Jobs, Praktika, Studium Bd. 60, interconnections Verlag, Freiburg.

Stark, Annika und Julian Weber (2012) Ehrt Euer chlorfreies Trinkwasser - Eine kritische Auseinandersetzung mit weltwärts-Blogs. In Berliner Entwicklungspolitischer Ratschlag (Hrsg.), Wer anderen einen Brunnen gräbt: Rassismuskritik, Empowerment, Globaler Kontext, Berlin.

Walter, Philipp (2012): Richtungswechsel: Ein weltwärts-Freiwilliger erzählt von seinen Erlebnissen und Erfahrungen in Namibia, Bloggingbooks, Saarbrücken. 